\title{
Guideline for Management of Mesenteric Ischemia: Shiraz Intestinal Failure Unit Protocol
}

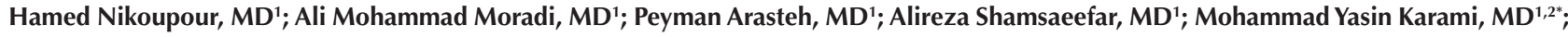 \\ Hesameddin Eghlimi, MD ${ }^{1}$; Mojtaba Shafiekhani, Pharm.D ${ }^{1,3}$; Saman Nikeghbalian; MD¹
}

${ }^{1}$ Shiraz Transplant Research Center, Shiraz University of Medical Sciences, Shiraz, Iran

${ }^{2}$ Department of Surgical Oncology, Shiraz University of Medical Sciences, Shiraz, Iran

${ }^{3}$ Department of Clinical Pharmacy, School of Pharmacy, Shiraz University of Medical Sciences, Shiraz, Iran

\begin{abstract}
We have recently established an intestinal rehabilitation unit (IRU) in Abu Ali Sina transplantation center affiliated to Shiraz University of Medical Sciences, Iran. Our intestinal failure rehabilitation and transplant program aims to provide state-of-the-art care for adult patients with different degrees of intestinal insufficiency and failure. In the IRU, we aimed to design an algorithmic approach to patients with small bowel ischemia and short bowel syndrome (SBS) based on our institutional experience in our country and based on other pioneering studies from other regions of the world.

Keywords: Disease management, Guideline, Mesenteric ischemia, Short Bowel Syndrome

Cite this article as: Nikoupour H, Moradi AM, Arasteh P, Shamsaeefar A, Karami MY, Eghlimi H, et al. Guideline for management of mesenteric ischemia: Shiraz intestinal failure unit protocol. Arch Iran Med. 2020;23(6):422-425. doi: 10.34172/aim.2020.38.
\end{abstract}

Received: October 20, 2019, Accepted: February 4, 2020, ePublished: June 1, 2020

\section{Introduction}

The most common mechanism of intestinal failure is short bowel syndrome (SBS). Furthermore, SBS is the main cause of intestinal failure in an estimated $75 \%$ and $50 \%$ of adults and pediatrics receiving parenteral nutrition (PN) in Europe. ${ }^{1,2}$ The condition is defined as loss in length of small intestine below a minimum required for adequate absorption of nutrients for appropriate growth and health, which may be caused by surgery or maybe due to congenital causes. ${ }^{3}$ More commonly in adults, symptoms related to SBS manifest when the intestine is shorter than $200 \mathrm{~cm}^{4}$

The leading cause of SBS is considered to be mesenteric vascular disease among adults and gastroschisis among pediatrics. ${ }^{5,6}$ Although the exact incidence and prevalence of SBS are not well understood, ${ }^{7}$ without proper management and planning, the condition is associated with high rates of mortality. Accordingly, the management of SBS has undergone significant alterations during past years. ${ }^{8}$

In the Middle East and most middle- and low-income countries, facilities for home parenteral nutrition (HPN) are largely missing; ${ }^{9}$ thus, management of patients with SBS is more intricate in these countries.

To this date, no specific complete algorithm or approach has been introduced, especially in countries where facilities for HPN are limited or non-existing. In this study, we aimed to design an algorithmic approach to patients with mesenteric ischemia and SBS based on our institutional experience in our country and based on other pioneering studies from other regions of the world.

\section{Material and Methods}

Historically, there have been different management approaches when facing a gangrenous bowel due to the lack of facilities for HPN in Iran. These have included: 1) leaving the gangrenous bowel in the abdominal cavity and waiting for the patient to die; 2) resection and primary anastomosis; which leads to anastomotic leakage in most cases.; and 3) jejunostomy tube insertion; which may cause electrolyte imbalance due to short bowel segment from the ligament of Treitz which would finally lead to death.

Due to limited options and the lack of facilities for HPN, most surgeons in Iran preferred resection and anastomosis, which led to unfavorable outcomes during past years. So, as the first small bowel rehabilitation and transplantation center in Iran, we designed a comprehensive management guideline when facing this entity in different settings.

Shiraz Intestinal Failure Protocol: in a Local Hospital As the clinical presentation of mesenteric ischemia is somehow confounding, abdominal pain and physical examinations are not conclusive and occasionally, the patient may be misdiagnosed as having appendicitis. ${ }^{10,11}$

When facing a gangrenous segment of the bowel (Figure 1), the following steps should be taken:

1- Measuring the length of the viable segments,

2- Resecting the gangrenous segment, 
3- Using a LigaSure device for dividing bowel meso, to minimize future adhesions,

4- Measuring the remnant jejunal segment from the ligament of Treitz.

Whenever the length of the jejunal remnant from the ligament of Treitz is more than $100 \mathrm{~cm}$, an end ostomy should be performed for the proximal segment; the distal viable segment which could be the distal section of the ileum or colon (mostly mid transverse colon) should then be stapled and closed and put inside the abdominal cavity.

In cases where the proximal jejunal remnant is shorter than $100 \mathrm{~cm}$, as this group of patients almost always require long-term PN support in hospital for preparation and bridging to small bowel reconstructive surgeries such as elongation surgeries, the jejunal remnant end should be stapled and closed. Meanwhile, for proximal decompression, a gastroduodenostomy tube should be inserted through the stomach and the distal viable bowel should be closed as mentioned in previous sections.

In both conditions, if the ileal remnant is longer than 50 $\mathrm{cm}$, it is better to perform stoma. However, in conditions where the ileal remnant is shorter than $50 \mathrm{~cm}$, it would be wise to staple and close it and put it in the abdominal cavity, taking into consideration that for future reconstructions, we may lose $5-10 \mathrm{~cm}$ of small bowel stock at the stoma site.

5- Checking coagulation factors: e.g. factor V Leiden, proteins $\mathrm{C}$ and $\mathrm{S}$, and performing echocardiography for evaluation of clot within the atrium and starting anticoagulation based on the patient's weight,

6- Preparing the patient for safe transportation to the intestinal rehabilitation unit (IRU) in stable conditions and with proper documentations and medical records.

Figure 1 shows the proposed management algorithm for small bowel ischemia.

\section{Shiraz Intestinal Failure Protocol: in the Intestinal Rehabilitation Unit}

In the IRU, as a special ward for small bowel support and rehabilitation, in patients with more than $100 \mathrm{~cm}$ of small bowel and stoma, special consideration should be given to avoid dehydration and electrolyte imbalance as follows: 1- Nutritional consideration
a. High salt diet,
b. Low fat - low sugar diet,
c. High carbohydrate intake,
d. Oral rehydration salts (ORS) instead of hypo- osmolar fluids.

2- Oral intake of Loperamide

After full stabilization of the patient, which usually takes about 1 week or more, the patient can be discharged from the hospital with close follow-up and regular outpatient visits, and may be planned for reconstructive surgeries three months later.

Patients with shorter than $100 \mathrm{~cm}$ of small bowel are hospitalized and planned for PN until their next surgical intervention. The patients' oral intake depends on the length of the distal ileal remnant: those with more than $50 \mathrm{~cm}$ of distal ileal segment who had an ostomy can start enteral feeding by inserting a catheter in the lumen of the bowel through the stoma with the goal of lowering the volume of PN.

After 2 months of proper management considering

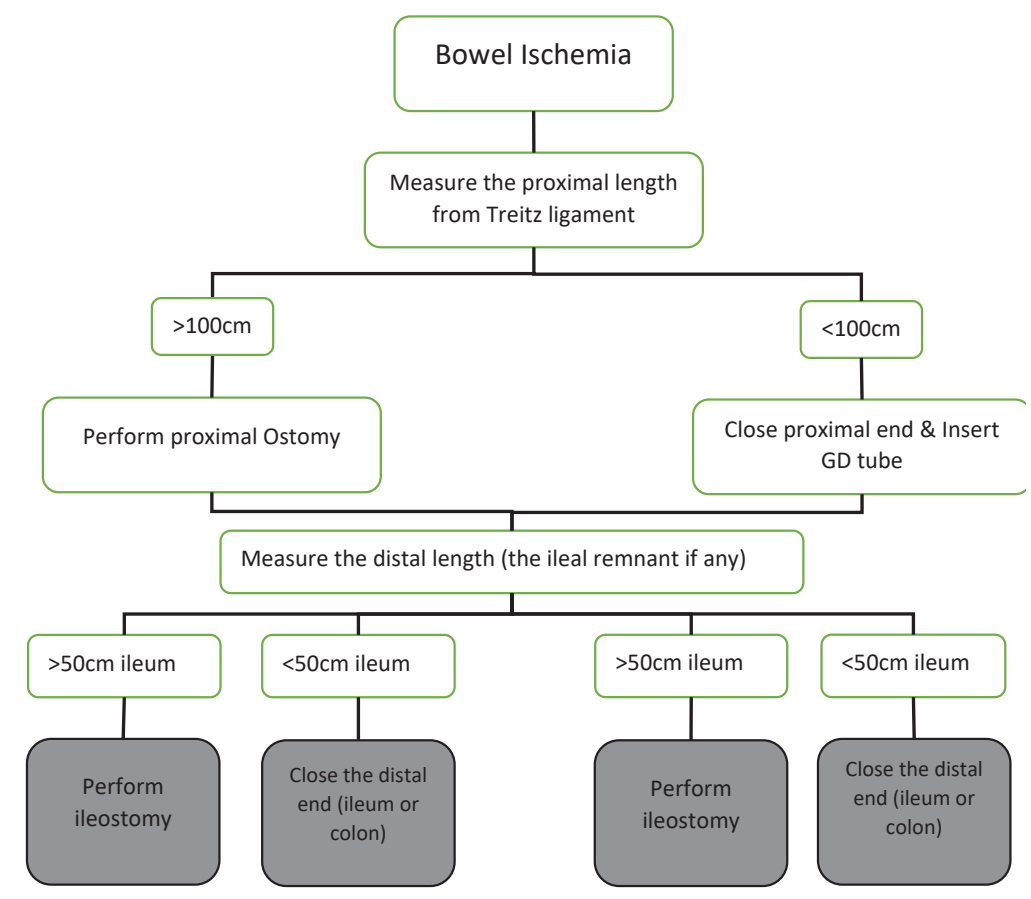

Figure 1. Algorithm for Management of Small Bowel Ischemia. Solid lines indicate accepted management plan. GD, gastroduodenal. 
essential nutritional needs and resolving the probable complications of PN and patient-related morbidities, the patients may become a candidate for autologous reconstructions, which depends on preoperative evaluations. These include contrast studies in order to determine the length and diameter of the bowel remnant and full discussions in a multi-disciplinary team (MDT) to define the best plan for the patient.

The MDT consists of transplant surgeons, gastroenterologists, nutritionists and intensive care unit specialists.

The future plan for these patients is based on the following considerations:

1- The aim of the surgery is to gradually wean the patient off $\mathrm{PN}$ or to prepare them for an upcoming small bowel transplantation

2- When preparing a patient to wean off PN, the bowel length is important as explained below:

a. In presence of the ileocecal valve (ICV), the minimum required length for the small bowel is 20-30 cm.

b. In the absence of the ICV but the presence of half of the length of the colon, the minimum required length for the small bowel would be $60-80 \mathrm{~cm}$. c. In the absence of the colon, patients should have at least $100 \mathrm{~cm}$ of bowel to expect a possibility of weaning off $\mathrm{PN}$.

The role of the ICV has been a subject of much controversy; however, it is widely accepted that the existence of the ICV improves chances for adaptation in patient with SBS. ${ }^{6,12}$

The goal of autologous gastrointestinal reconstruction (AGIR) surgery is to obtain this minimum length for the small bowel.

In almost all patients, abdominal fascia will be left open and only the skin would be closed. Since the length of the small bowel remnant is much less than its native and due to postoperative adhesions, abdominal protrusion occur less.

Using mesh grafts for fascia closure may increase the rate of infection. On the other hand, leaving it open allows drainage of intra-abdominal fluid and will lower the rate of collections. All intestinal anastomoses are performed as hand sewn in two layers.

Figure 2 shows the proposed management algorithm for autologous gastrointestinal reconstruction.

After the surgeries, PN will continue until the patient is able to take $60 \%$ of their necessary daily calories via the

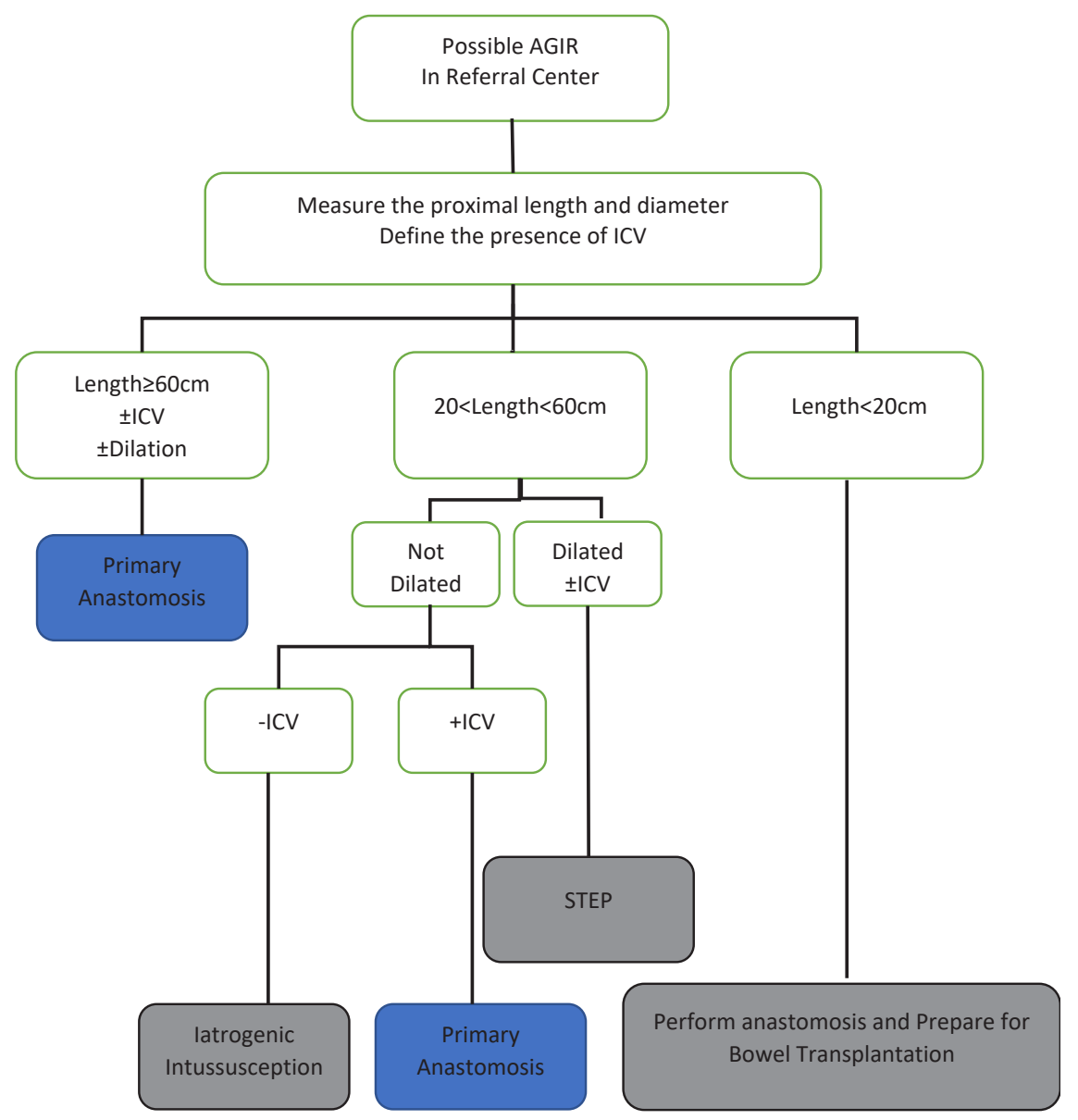

Figure 2. Algorithm for Autologous Gastrointestinal Reconstruction. AGIR, autologous gastrointestinal reconstruction; ICV, ileocecal valve; STEP, serial transvers elongation procedures. 


\section{PO route.}

For the patient to become a candidate for serial transverse enteroplasty (STEP) procedure, the minimum length and diameter of the remnant segment of small bowel should be $20 \mathrm{~cm}$ and $4 \mathrm{~cm}$, respectively.

Gastroduodenostomy tube will be kept in place and will be removed in follow-up visits.

Patients with ultra-short bowels (less than $20 \mathrm{~cm}$ in length $)^{13}$ should be listed for small bowel transplantation and all related work-up should be done. Furthermore, these patients should all have an established continuity of bowel; in cases where continuity of bowel does not exist, the patients should undergo surgery for establishment of bowel continuity. Accordingly, mortality among these patients will decrease. Moreover, by performing AGIR surgeries, the possible need for bowel transplantation will also decrease.

In conclusion, we reported here our experience and clinical algorithm when facing patients with SBS in a setting where limited or no HPN exists.

\section{Authors' Contribution}

HN, AMM and MYK: Hypothesis, manuscript preparation and final revision. PA: Hypothesis and manuscript preparation. AS, HE and MS: Hypothesis and final revision. SN: Hypothesis, supervision and final revision.

\section{Conflict of Interest Disclosures}

The authors have no conflicts of interest.

\section{Ethical Statement}

This study was approved by the ethical committee of Shiraz University of Medical Sciences.

\section{References}

1. Squires RH, Duggan C, Teitelbaum DH, Wales PW, Balint J, Venick R, et al. Natural history of pediatric intestinal failure: initial report from the Pediatric Intestinal Failure Consortium. J Pediatr. 2012;161(4):723-8.e722. doi: 10.1016/j. jpeds.2012.03.062.

2. Pironi L, Goulet O, Buchman A, Messing B, Gabe S, Candusso $\mathrm{M}$, et al. Outcome on home parenteral nutrition for benign intestinal failure: a review of the literature and benchmarking with the European prospective survey of ESPEN. Clin Nutr. 2012;31(6):831-45. doi: 10.1016/j.clnu.2012.05.004.

3. Batra A, Keys SC, Johnson MJ, Wheeler RA, Beattie RM. Epidemiology, management and outcome of ultrashort bowel syndrome in infancy. Arch Dis Child Fetal Neonatal Ed. 2017;102(6):F551-6. doi:10.1136/archdischild-2016-311765.

4. Lauro A, Cirocchi R, Cautero N, Dazzi A, Pironi D, Di Matteo $\mathrm{FM}$, et al. Reconnection surgery in adult post-operative short bowel syndrome $<100 \mathrm{~cm}$ : is colonic continuity sufficient to achieve enteral autonomy without autologous gastrointestinal reconstruction? Report from a single center and systematic review of literature. G Chir. 2017;38(4):163. doi: 10.11138/ gchir/2017.38.4.163.

5. Wang M-Y, Wu M-H, Hsieh D-Y, Lin LJ, Lee PH, Chen WJ, et al. Home parenteral nutrition support in adults: experience of a medical center in Asia. JPEN J. 2007;31(4):306-10. doi: 10.1177/0148607107031004306.

6. Lauro A, Lacaille F. Short bowel syndrome in children and adults: from rehabilitation to transplantation. Expert Rev Gastroenterol Hepatol. 2019;13(1):55-70. doi: 10.1080/17474124.2019.1541736

7. Guillen B, Atherton NS. Short Bowel Syndrome. Treasure Island (FL): StatPearls Publishing; 2020.

8. Cohran VC, Prozialeck JD, Cole CR. Redefining short bowe syndrome in the 21st century. Pediatr Res. 2017;81(4):540-9. doi: 10.1038/pr.2016.265.

9. Gondolesi GE, Pattin F, Nikkoupur H. Management of intestinal failure in middle-income countries, for children and adults. Curr Opin Organ Transplant. 2018;23(2):212-8. doi: 10.1097/MOT.0000000000000512.

10. Tilsed J, Casamassima A, Kurihara H, Mariani D, Martinez I, Pereira J, et al. ESTES guidelines: acute mesenteric ischaemia. Eur J Trauma Emerg Surg. 2016;42(2):253-70. doi: 10.1007/ s00068-016-0634-0.

11. Luther B, Mamopoulos A, Lehmann C, Klar E. The ongoing challenge of acute mesenteric ischemia. Visc med. 2018;34(3):215-21. doi: 10.1159/000490318.

12. Kaji T, Nakame K, Machigashira S, Kawano T, Masuya R, Yamada W, et al. Predictors of a successful outcome for infants with short bowel syndrome: a 30-year single-institution experience. Surg today. 2017;47(11):1391-6. doi: 10.1007/ s00595-017-1534-6.

13. Pironi L. Definitions of intestinal failure and the short bowe syndrome. Best Pract Res Clin Gastroenterol. 2016;30(2):17385.doi: 10.1016/j.bpg.2016.02.011. 